\title{
BIOLOGIA FLORAL E SISTEMA DE POLINIZAÇÃO DE SOLANUM STRAMONIFOLIUM JACQ. (SOLANACEAE) EM REMANESCENTE DE MATA ATLÂNTICA, PERNAMBUCO ${ }^{1}$
}

\author{
Elisangela Lúcia de S. Bezerra² \\ Isabel Cristina Machado ${ }^{3}$
}

Recebido em 20/01/02. Aceito em 30/08/2002

\begin{abstract}
RESUMO - (Biologia floral e sistema de polinização de Solanum stramonifolium Jacq. (Solanaceae) em remanescente de Mata Atlântica, Pernambuco). A deiscência de anteras através de pequenos poros apicais é uma característica encontrada em várias espécies da família Solanaceae, especialmente no gênero Solanum, característica esta que restringe a polinização a um grupo de abelhas fêmeas capazes de vibrar as anteras para retirada do pólen. A fenologia, biologia floral e a polinização de Solanum stramonifolium foram estudadas entre os meses de agosto/1999 a dezembro/2000 em populações naturais ocorrentes no Parque Estadual de Dois Irmãos, um dos poucos remanescentes de Mata Atlântica localizada em Recife, Pernambuco (87'30'S e 3452'30"W). Solanum stramonifolium é um arbusto com flores dispostas em inflorescências racemosas, apresentando corola branca e cinco estames de um amarelo intenso, com anteras poricidas dispostas ao redor do gineceu. A antese ocorre nas primeiras horas do dia, havendo reflexão de luz ultravioleta e presença de áreas de concentração de emissão de odor por toda a corola e na região apical das anteras. Solanum stramonifolium tem padrão de floração contínuo e apresenta flores hermafroditas $(62 \%)$ e funcionalmente masculinas $(38 \%)$, o que caracteriza a espécie como andromonóica. Treze espécies de abelhas foram observadas visitando as flores de S. stramonifolium (nove polinizadores e quatro pilhadores). A constante e grande produção de flores faz com que Solamun stramonifolium mantenha sua guilda de polinizadores e garanta assim a sua reprodução, sendo, ao mesmo tempo, uma importante fonte de recursos para a manutenção destas abelhas.
\end{abstract}

Palavras-chave - Solanum, polinização vibrátil, andromonoicia, Floresta Atlântica, melitofilia

\begin{abstract}
Floral biology and pollination system of Solanum stramonifolium Jacq. (Solanaceae) in an Atlantic Forest remnant in Pernambuco). The anthers deiscense through two small apical pores is a feature found in many species of Solanaceae, especially in the genus Solanum. This feature restricts pollination to a group of female bees which are able to vibrate the anthers (buzz pollination). The phenology, floral biology and pollination of Solanum stramonifolium were studied between August/1999 and December/2000 in natural populations occurring at Dois Irmãos State Park, one of the last Atlantic forest remnants in Recife city, Pernambuco state
\end{abstract}

1 Trabalho vencedor do Concurso Prêmio Verde concedido pela Sociedade Brasileira de Botânica do Brasil (SBB/2001).

Bolsista PIBIC/CNPq/UFPE (elsbezerra@zipmail.com.br)

3 Departamento de Botânica, CCB, Universidade Federal de Pernambuco, Av. Prof. Nelson Chaves s/n, Cidade Universitária, CEP 50372-970, Recife, PE, Brasil (imachado@ufpe.br) 
( $8^{\circ} 7^{\prime} 30^{\prime \prime} \mathrm{S}$ and $\left.34^{\circ} 52^{\prime} 30^{\prime \prime} \mathrm{W}\right)$. Solanum stramonifolium is a shrub whose flowers are arranged in a racemose inflorescence and have white corolla and five stamens with poricide anthers disposed around the gynoecium. Anthesis occur early in the morning and the petals present a ultraviolet reflection pattern. Solanum stramonifolium presents continuous flowering pattern and have hermaphrodite (62\%) and functional male flowers (38\%), therefore the species is andromonoicious. Thirteen species of bees were observed visiting the flowers of S. stramonifolium (nine pollinators and four pollen robbers). The great and constant production of flowers of S. stramonifolium along the year is important to maintain the pollinators guild and to guarantee the plant reproduction.

Key words - Solanum, buzz-pollination, Atlantic Forest, melittophily, andromonoicy

\section{Introdução}

A deiscência de anteras através de pequenos poros apicais é uma característica marcante em várias espécies da família Solanaceae, especialmente do gênero Solanum. Dentre as 15.000 ou até 20.000 espécies dos 544 gêneros e 72 famílias de Angiospermas que apresentam flores com anteras poricidas (Buchmann 1983), o gênero Solanum compreende mais de 1.400 espécies com distribuição cosmopolita e preferencialmente tropical (D'Arcy 1973), tendo sido objeto de vários estudos em biologia floral (Harris \& Kucks 1902; Linsley 1962; Linsley \& Cazier 1963; D'Arcy 1973; Bowers 1975; Buchmann et al. 1977; Schilling \& Heiser 1979; Oliveira \& Oliveira 1988; Avanzi \& Campos 1997).

A polinização em espécies com anteras poricidas é feita por algumas abelhas fêmeas que são hábeis na coleta do pólen, através da vibração das anteras. Esse comportamento é chamado de "buzz-pollination" ou polinização por vibração (Buchmann 1983).

As três categorias de abelhas que visitam flores com anteras poricidas descritas por Michener (1962), Wille (1963) e Buchmann et al. (1977): 1 - abelhas vibráteis, 2 - abelhas mordedoras e 3 - abelhas coletoras, com a subcategoria "milking" (espremedoras), são encontradas visitando flores do gênero Solanum. Algumas efetuando a polinização e outras apenas pilhando.

Solanum stramonifolium Jacq. engloba todas as características de flores polinizadas por vibração e, neste trabalho, são apresentados estudos sobre a morfologia, biologia floral e fenologia desta espécie de Solanum, com ênfase nos mecanismos de polinização e nas interações planta-polinizador.

\section{Material e métodos}

Os estudos sobre a fenologia, biologia floral e o sistema de polinização de $S$. stramonifolium foram desenvolvidos entre agosto/1999 a dezembro/2000 em agrupamentos naturais constituídos de poucas plantas esparsas, até agrupamentos com mais de 40 indivíduos, distribuídos na borda da mata, localizada no Parque Estadual de Dois Irmãos ( $8^{\circ} 7^{\prime} 30^{\prime \prime} \mathrm{S}$ e 3452'30”W), região metropolitana do Recife, Pernambuco, um dos poucos remanescentes de Mata Atlântica do Estado.

O Parque possui cerca de 370 ha e está distribuído em relevo levemente ondulado, com altitude de 30 a $80 \mathrm{~m}$, onde as precipitações pluviométricas atingem, em média, anualmente, cerca de $2.000 \mathrm{~mm}$. A temperatura do ar tem como valores mínimos $24^{\circ} \mathrm{C}$, nos meses de junho a agosto, e valores máximos de $27^{\circ} \mathrm{C}$, de dezembro a março (Machado et al. 1998).

Foram feitas observações sobre a morfologia, tamanho, coloração, duração e odor das flores, horário, duração e sequiência da antese e receptividade do estigma (Dafni 1992; Kearns \& Inouye 1993), diretamente no campo e complementadas através da análise de desenhos esquemáticos realizados no laboratório, com o auxílio de câmara-clara acoplada a estereomicroscópio. Medidas acerca do diâmetro da corola de flores grandes e 
pequenas foram realizadas a fim de se verificar diferenças significativas utilizando teste $\mathrm{t}$ através do software BioEstat 1.0.

$\mathrm{O}$ percentual dos diferentes tipos florais com relação à sexualidade foi determinado a partir da análise de 1.273 inflorescências e aproximadamente 1.200 flores num agrupamento com 12 indivíduos. Foram feitas também observações dos aspectos da fenologia da floração e frutificação através do acompanhamento semanal de dez indivíduos marcados aleatoriamente no campo. No decorrer de um ano, todas as inflorescências foram marcadas com etiquetas plásticas, sendo registrados o número de flores e o tipo floral, o número de frutos e de botões. O padrão de floração da espécie foi determinado a partir dos modelos apresentados por Gentry (1974) e Newstrom et al. (1994).

As áreas de concentração de emissão de odor foram detectadas através de solução aquosa de vermelho neutro (Vogel 1990) e a análise referente à absorção e reflexão de luz ultravioleta foi realizada através de observação direta de flores frescas em câmara provida com luz ultravioleta (Buchmann et al.1977).

Para analisar a carga e a disponibilidade polínica, foram ensacadas 12 inflorescências, das quais foram coletados dez botões em préantese, dez flores após 3h de exposição aos visitantes, dez flores com $6 \mathrm{~h}$ de exposição e outras dez após 9 h de início da antese. O número de grãos de pólen por antera foi estimado utilizando-se uma câmara de Neubauer (Moura et al. 1987).

A fenologia e o comportamento dos visitantes foram estudados a partir de observações visuais diretas no campo em horários variados do dia, em um agrupamento com 15 indivíduos, e complementados pela análise de fotografias obtidas em campo. Durante o período de desenvolvimento do estudo, 50 horas de observação, distribuídas em oito dias entre os meses de novembro e dezembro/2000, das 5:00h às 15:00h, foram dedicadas exclusivamente para o registro das abelhas durante suas visitas. Neste período foi feita a classificação das abelhas raras, comuns e muito comuns, de acordo com a freqüência de visitas, tendo como critério o percentual obtido com as observações (de 0 a $5 \%$, de 5 a $15 \%$ e de 15 a $45 \%$, respectivamente). Quanto ao tamanho, os visitantes foram classificados (sensu Frankie et al.1983) como grandes (comprimento igual ou superior à $12 \mathrm{~mm}$ ) e pequenos (menos de $12 \mathrm{~mm}$ de comprimento).

Algumas abelhas foram capturadas para verificar o local de deposição de pólen e para posterior identificação por especialistas. Os indivíduos coletados foram conservados a seco e encontram-se na coleção do Laboratório de Biologia Floral e Reprodutiva da Universidade Federal de Pernambuco, como espécimestestemunho.

Espécime-testemunho de Solanum stramonifolium foi depositado no Herbário UFP - Professor Geraldo Mariz, Universidade Federal de Pernambuco (UFP 27.719).

\section{Resultados e discussão}

Solanum stramonifolium é um arbusto perene encontrado em bordas de mata e com comportamento de espécie pioneira. Suas flores estão dispostas em inflorescências racemosas, pentâmeras, actinomorfas, com cinco anteras poricidas, apresentando em média duas a três flores abertas, abrindo-se pelo menos uma por dia em cada inflorescência. $\mathrm{Na}$ fase de botão floral, as pétalas apresentam cor lilás em sua face externa, enquanto durante a antese a corola é branca, com cinco anteras de um amarelo vivo, dispostas ao redor do estilete (Fig. 1). Além de suas cores visíveis, as flores possuem um padrão de reflexão de ultravioleta concentrado no ápice das pétalas e no pedicelo das flores que, ao longo da antese, vai sendo perdido.

As flores de $S$. stramonifolium apresentam atributos que as enquadram na síndrome da melitofilia, descrita por Faegri \& Pijl (1979) e, 

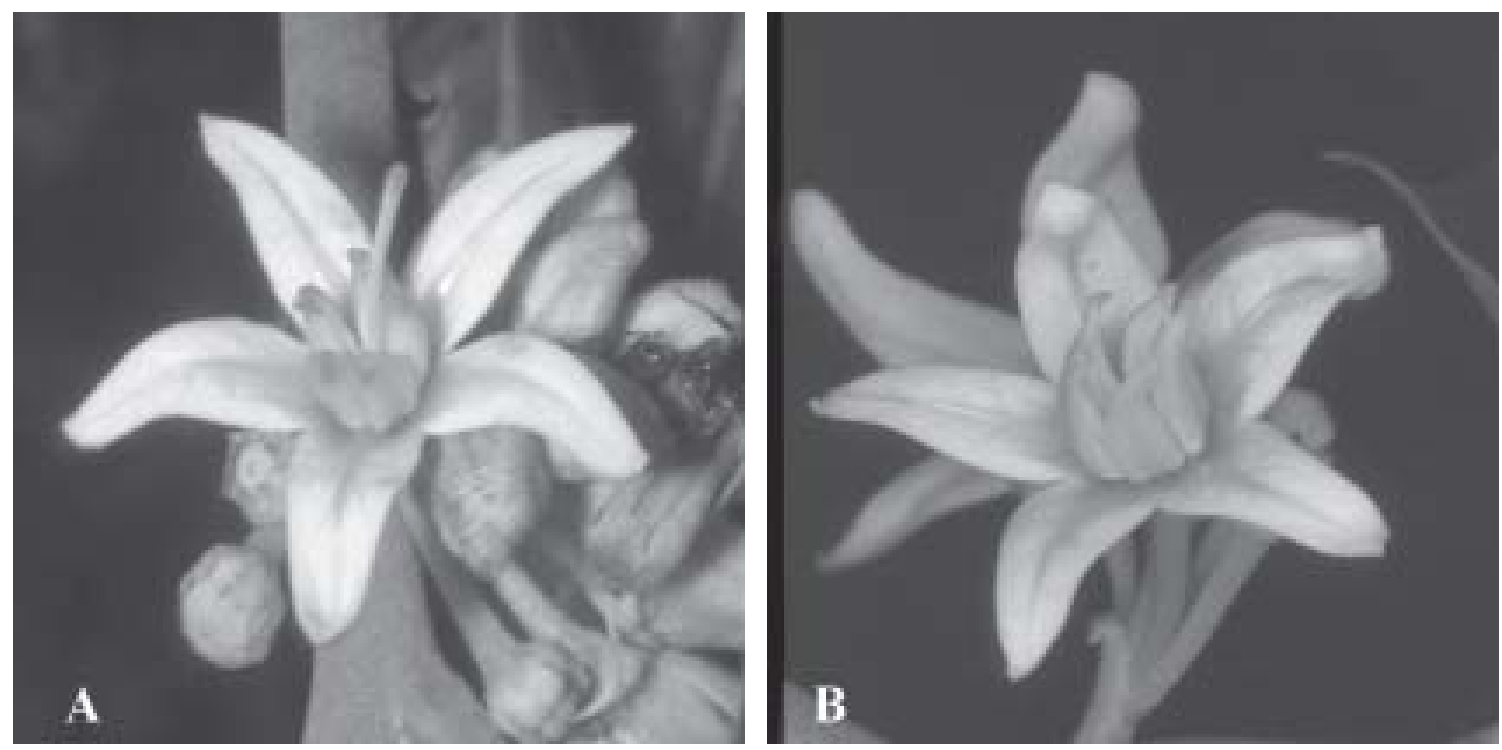

Figura 1. Flores de Solanum stramonifolium. A) flor de estilete longo; B) flor de estilete curto.

em especial, na síndrome de polinização vibrátil descrita por Buchmann (1983), tais como: antese diurna; ornamentação da corola com padrões visíveis e de ultravioleta contrastantes; presença de áreas de concentração de emissão de odor; estames coloridos, vistosos; anteras poricidas coniventes ao redor do estilete, com grãos de pólen pequenos e leves, liberados por vibração mecânica direta. Os grãos são lisos e sem adesivos lipídicos, o que facilita a sua liberação.

Esta combinação de características e a deiscência das anteras definem a síndrome de polinização por vibração ("buzz pollination”) para o gênero Solanum (Harris \& Kucks 1902; Linsley 1962; Linsley \& Casier 1963; Bowers 1975; Buchmann et al.1977; Buchmann 1983; Vogel 1978; Schilling \& Heiser 1979; Coleman \& Coleman 1982; Endress 1994). Em seu trabalho, Dukas e Dafni (1990) sugerem que as flores polinizadas por movimentos vibratórios evoluíram de flores poliândricas nectaríferas que a priori reduziram o número de estames (e conseqüentemente a produção de pólen) seguida de redução da produção de néctar. No caso do gênero Solanum, as flores são isostêmones, caracterizando um tipo floral, denominado Solanum (Vogel 1978; Bernhardt 1996).

A única recompensa floral oferecida por S. stramonifolium é o pólen, que é coletado exclusivamente por abelhas fêmeas que utilizam o recurso na alimentação de suas larvas. A procura destes animais por flores que oferecem este tipo de recurso, levou, ao longo da evolução floral, ao surgimento das flores de pólen ("pollen flowers"), as quais não secretam néctar ou qualquer outro tipo de recurso floral (Vogel 1978; Bernhardt 1996).

A antese inicia em horários variados ao longo do dia, estando a maioria das flores abertas às 5:00h e outras no decorrer do dia, até o entardecer. As flores permanecem abertas na planta por cerca de $36 \mathrm{~h}$, durando algumas vezes até 48 h. Com o passar do tempo, as anteras vão se desgastando e ficando amarronzadas. A manutenção das flores por mais de um dia é vantajosa para a planta por ser energeticamente menos dispendioso mantê-las, reduzindo assim, à metade a produção de flores novas todos os dias. Desta forma, as flores velhas funcionam como um "display" visual para as abelhas às flores de Solanum. Outro atrativo visual é a 
absorção e reflexão de luz ultravioleta como também é visto em $S$. sessiliflorum (Storti 1988), S. lycocarpum (Oliveira \& Oliveira 1988), S. douglasii e S. xanti (Buchmann et al. 1977). As flores tratadas em solução de vermelho neutro revelaram áreas de concentração de emissão de odor próximas às margens da corola e algumas esparsas por todo o perianto. Em relação ao estigma, apenas o das flores de estilete longo apresentaram-se corados.

S. stramonifolium apresenta dois tipos florais, classificados a partir do comprimento de estilete e da função desempenhada: a) flores de estilete longo, hermafroditas (Fig. 1A) e b) flores de estilete curto (Fig. 1B), nas quais observa-se uma gradação em relação ao comprimento do estilete sendo, porém, funcionalmente masculinas. Apenas no primeiro tipo floral, o estilete exterioriza-se além do cone de anteras. Há também flores com diferentes dimensões, grandes e pequenas $(\mathrm{p}<0.001)$, com diâmetro médio da corola de $2,74 \mathrm{~cm}$ (DP = $\pm 0.0026)$ e $1,68 \mathrm{~cm}(\mathrm{DP}= \pm 0.0008)$ respectivamente, ambas ocorrendo no mesmo indivíduo e até na mesma inflorescência, expostas à luz ou em áreas sombreadas. Também foram encontradas, esporadicamente, flores trímeras, tetrâmeras e outras unicamente masculinas. O estigma dos dois tipos florais encontra-se receptivo durante toda a duração da flor. A apresentação de diferentes tipos florais é bastante comum no gênero Solanum (Symon 1979), sendo também observado nas espécies S. sessiliflorum (Storti 1988), S. lycocarpum (Oliveira \& Oliveira 1988), S. aculeatissimum e S. variabile (Avanzi \& Campos 1997), nas quais apenas as flores de estilete longo formam fruto. A produção de flores pequenas pode ser uma estratégia desta espécie de Solanum para atrair as pequenas abelhas vibradoras que polinizam outras espécies simpátricas do gênero, visto que essas abelhas são mais eficientes nestas flores devido à redução da distância entre o estigma e a antera (Buchmann et al. 1977), abraçando diretamente todo o cone estaminal com seu curto tórax.

Solanum stramonifolium apresenta padrões de floração e frutificação contínuos, com pico entre os meses de setembro a dezembro e outubro a fevereiro, respectivamente. Foi encontrado número médio de 20 botões por inflorescência, predominando as flores de estilete longo em todas as inflorescências de todos os indivíduos, totalizando $62 \%$, das quais $93 \%$ formam fruto sob condições naturais. A fenologia da floração de $S$. stramonifolium, com padrão de floração contínuo (sensu Newstrom et al. 1994; Gentry 1974) e a produção de flores funcionalmente masculinas observadas em S. stramonifolium (38\%) garantem a constância e frequiência das visitas dos polinizadores.

Logo após a abertura das flores, grande quantidade de pólen é liberada através da ação dos visitantes que, nas primeiras três horas de visita, retiram aproximadamente $40 \%$ da quantidade total de pólen, permanecendo, após seis horas de abertura da flor, apenas $12 \%$ do total de grãos (Fig. 2). Contudo, no final da manhã ou ao entardecer, quando o recurso é escasso ou inexistente, as flores continuavam a receber visitas, sendo agora, porém submetidas a uma quantidade maior de movimentos de vibração pelas abelhas. A diminuição do recurso

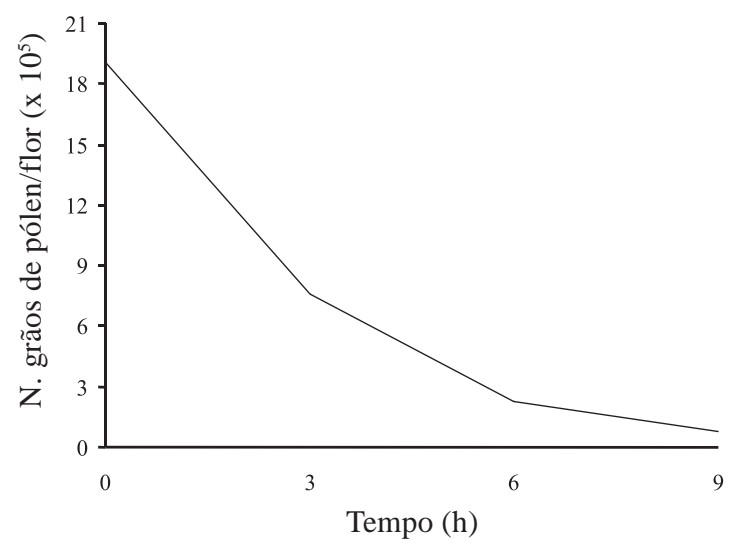

Figura 2. Disponibilidade polínica de Solanum stramonifolium. 
acarreta, portanto, alteração no comportamento de coleta de pólen pelas abelhas, que passam então a girar repetidas vezes sobre a mesma flor, bem como induz a um número maior de visitas a flores, como descrito por Buchmann (1983). A alteração da cor da corola com a idade das flores, assim como o ressecamento do ápice, a conseqüente perda do amarelo vivo das anteras, constituem indicadores da redução da disponibilidade do recurso, semelhante ao observado em S. lycocarpum (Oliveira \& Oliveira 1988) .

Treze espécies de abelhas visitam as flores de S. stramonifolium (Tab. 1). Apenas alguns gêneros de abelhas têm a capacidade de promover a polinização vibrátil, entre eles, Augochloropsis, Bombus, Eufriesea, Euglossa, Eulaema, Melipona, Paratetrapedia, Pseudoaugochloropsis e Xylocopa (Avanzi \& Campos 1992; Lopes \& Machado 1986; Oliveira

Tabela 1. Visitantes florais de Solanum stramonifolium no Parque Estudual de Dois Irmãos, classificados de acordo com Roubik (1992).

\begin{tabular}{lcccc}
\hline Visitantes & $\begin{array}{c}\text { Comprimento } \\
\text { do corpo }(\mathrm{mm})\end{array}$ & $\begin{array}{c}\text { Comportamento/ } \\
\text { Categoria }\end{array}$ & $\begin{array}{c}\text { Duração } \\
\text { da vibração (seg) }\end{array}$ & $\begin{array}{c}\text { Freqüência } \\
\text { de visitas }\end{array}$ \\
\hline
\end{tabular}

\begin{tabular}{|c|c|c|c|c|}
\hline \multicolumn{5}{|l|}{ APIDAE } \\
\hline \multicolumn{5}{|l|}{ Bombinae } \\
\hline \multicolumn{5}{|l|}{ Bombini } \\
\hline Bombus brevivillus** Franklin, 1913 & $15-18$ & $\mathrm{Po} / \mathrm{Vi}$ & 5 & $\mathrm{R}$ \\
\hline \multicolumn{5}{|l|}{ Euglossini } \\
\hline $\begin{array}{l}\text { Eufriesea surinamensis** } \\
\quad \text { (Linnaeus, 1758) }\end{array}$ & $18-19$ & $\mathrm{Po} / \mathrm{Vi}$ & 3 & $\mathrm{MC}$ \\
\hline Euglossa sp.** & $11-13$ & $\mathrm{Po} / \mathrm{Vi}$ & 4 & $\mathrm{R}$ \\
\hline Eulaema cingulata $* *$ (Fabricius, 1804$)$ & $18-21$ & $\mathrm{Po} / \mathrm{Vi}$ & 3 & $\mathrm{R}$ \\
\hline Eulaema nigrita** Lepeletier (1841) & 23 & $\mathrm{Po} / \mathrm{Vi}$ & 5 & $\mathrm{R}$ \\
\hline \multicolumn{5}{|l|}{ Meliponinae } \\
\hline \multicolumn{5}{|l|}{ Meliponini } \\
\hline Melipona scutellaris** Latreille (1811) & $10-14$ & $\mathrm{Po} / \mathrm{Vi}$ & 5 & $\mathrm{MC}$ \\
\hline \multicolumn{5}{|l|}{ ANTHOPHORIDAE } \\
\hline \multicolumn{5}{|l|}{ Anthophorinae } \\
\hline \multicolumn{5}{|l|}{ Exomalopsini } \\
\hline Paratetrapedia sp.* & 5 & $\mathrm{Pi} / \mathrm{Vi}$ & 8 & $\mathrm{R}$ \\
\hline \multicolumn{5}{|l|}{ Trigonini } \\
\hline Plebeia sp.* & 4 & $\mathrm{Pi} / \mathrm{Co}$ & NV & $\mathrm{R}$ \\
\hline Tetragonisca angustula* (Latreille 1811) & 4 & $\mathrm{Pi} / \mathrm{Co}$ & NV & $\mathrm{R}$ \\
\hline Trigona sp.* & 5 & $\mathrm{Pi} / \mathrm{Mo}$ & NV & $\mathrm{R}$ \\
\hline \multicolumn{5}{|l|}{ Xylocopinae } \\
\hline \multicolumn{5}{|l|}{ Xylocopini } \\
\hline Xylocopa suspecta** & $24-26$ & $\mathrm{Po} / \mathrm{Vi}$ & 3 & $\mathrm{MC}$ \\
\hline \multicolumn{5}{|l|}{ Moure \& Camargo, 1988} \\
\hline \multicolumn{5}{|l|}{ HALICTIDAE } \\
\hline \multicolumn{5}{|l|}{ Halictinae } \\
\hline \multicolumn{5}{|l|}{ Augochlorini } \\
\hline Augochloropsis sp.* & $8-10$ & $\mathrm{Po} / \mathrm{Vi}$ & 9 & $\mathrm{C}$ \\
\hline Pseudoaugochloropsis sp.* & $10-12$ & $\mathrm{Po} / \mathrm{Vi}$ & 8 & $\mathrm{C}$ \\
\hline
\end{tabular}


\& Oliveira 1988). Espécies de todos esses gêneros foram observadas explorando as flores de Solanum stramonifolium como fonte de pólen necessária para o desenvolvimento de suas larvas. O acompanhamento fenológico desses insetos revela períodos onde predominam, em cada um deles, determinada espécie (Fig. 3). De janeiro ao início de março, Eulaema nigrita é o polinizador mais freqüente, e de meados de março até maio, foi encontrada maior atividade de Xylocopa suspecta. Do mês de maio até o final do ano, Melipona scutellaris supera todos os outros visitantes, chegando a realizar uma média de 171 visitas diárias no seu horário de maior atividade (Fig. 4). Os representantes da família Halictidae, que visitam as flores de S. stramonifolium, se encontraram presentes ao longo do ano. Entre os meses de outubro a dezembro, foi encontrada a maior diversidade de visitantes, sendo observadas todas as 13 espécies de abelhas visitando as flores de Solanum stramonifolium.

O acompanhamento fenológico dos visitantes possibilita concluir que, dependendo da época do ano, é possível encontrar diferentes espécies como sendo o polinizador mais eficiente em relação à freqüência das visitas e que o período de maior diversidade das abelhas coincide com o pico de floração da planta. Portanto, as flores de $S$. stramonifolium são recurso importante para a manutenção desta guilda de abelhas no ecossistema estudado.

Nas 50 horas de observação, entre os meses de novembro e dezembro/2000, Melipona scutellaris foi a abelha mais freqüente, seguida de Xylocopa suspecta, Eufriesea surinamensis, Pseudoaugochloropsis sp., Augochloropsis sp., Euglossa sp., Eulaema cingulata, Eulaema nigrita, Paratetrapedia sp. e Bombus brevivillus (Fig. 4). O tamanho das abelhas variou de 4 a 26mm (Tab. 1).

Melipona, Eufriesea e Xylocopa foram comumentes polinizadores matinais e crepusculares, com seus maiores períodos de atividade ocorrendo das 7:00h às 11:00h e de menor atividade, do meio-dia até o entardecer (Fig. 4).

Melipona scutellaris (Fig. 5A) aproxima-se da flor frontalmente e, ao pousar, vibra seu tórax sobre todo o cone de anteras. Nas flores velhas ou muito visitadas, esta abelha vibra fazendo movimentos giratórios sobre a flor $\mathrm{e}$, assim como as abelhas maiores, toca o estigma das flores de estilete longo com seu abdômen

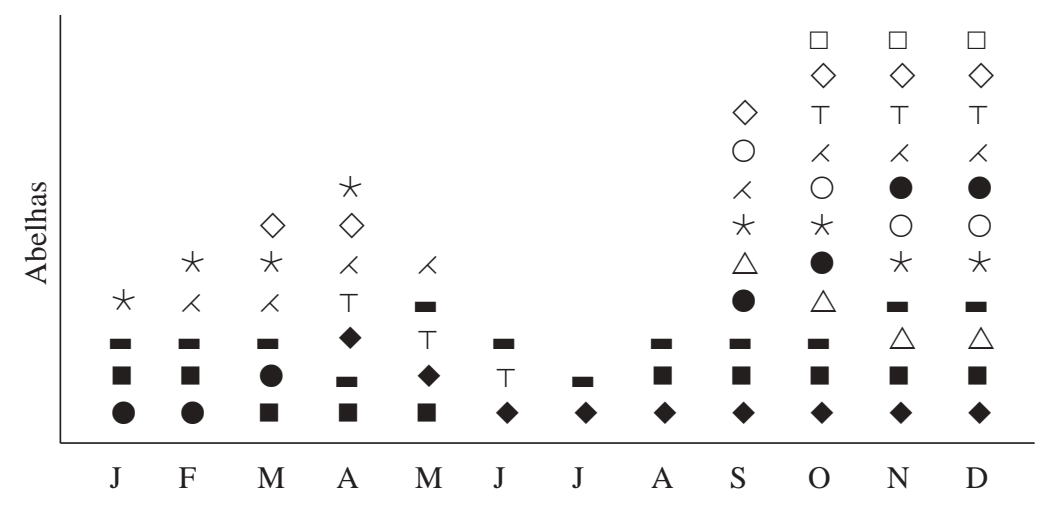

Figura 3. Acompanhamento fenológico das abelhas visitantes de Solanum stramonifolium de janeiro a dezembro/2000. As linhas verticais mostram que a cada mês as espécies de abelhas variam em relação à sua freqüência de visitas, estando as mais freqüentes, a cada mês, na base e as menos freqüentes no ápice. Melipona scutellaris, Xylocopa suspecta, $\triangle$ Eufriesea surinamensis; — Halictidae, $\star$ Euglossa sp., ○ Eulaema cingulata, $\bullet$ Eulaema nigrita, $\curlywedge$ Paratetrapedia sp., $\uparrow$ Bombus brevivilus, $\diamond$ Tetragonisca angustula, $\square$ Plebeia $\mathrm{sp.}$ 
cheio de pólen. Após a coleta, realiza comportamento de limpeza, pairando no ar, no intervalo entre as visitas, transferindo o pólen do tórax e do abdômen para as corbículas das pernas posteriores, com o auxílio das pernas anteriores e medianas. Em sua rota, visita quase que todas as flores de um mesmo indivíduo, deslocando-se, em seguida, para outro indivíduo.

Assim como Melipona, as demais abelhas grandes (Tab. 1) vibram todo o cone de anteras, liberando, neste momento, uma nuvem de pólen. Em sua rota, Xylocopa suspecta (Fig. 5B) visita em média 18 flores. Eufriesea surinamensis,
Eulaema cingulata, E. nigrita e Bombus brevivillus visitam cerca de 20 flores e Euglossa sp., cerca de oito. A aproximação destas abelhas também é frontal e a transferência de pólen para o armazenamento nas corbículas é feita em vôo, defronte à flor visitada. Com a diminuição da quantidade de pólen, ao longo do dia, estas abelhas passam a girar em cima do cone das anteras, procurando uma posição para efetivar com maior eficiência a coleta do pólen.

As abelhas pequenas que vibram (Pseudoaugochloropsis sp., Augochloropsis sp. e Paratetrapedia sp.) abordam diretamente cada

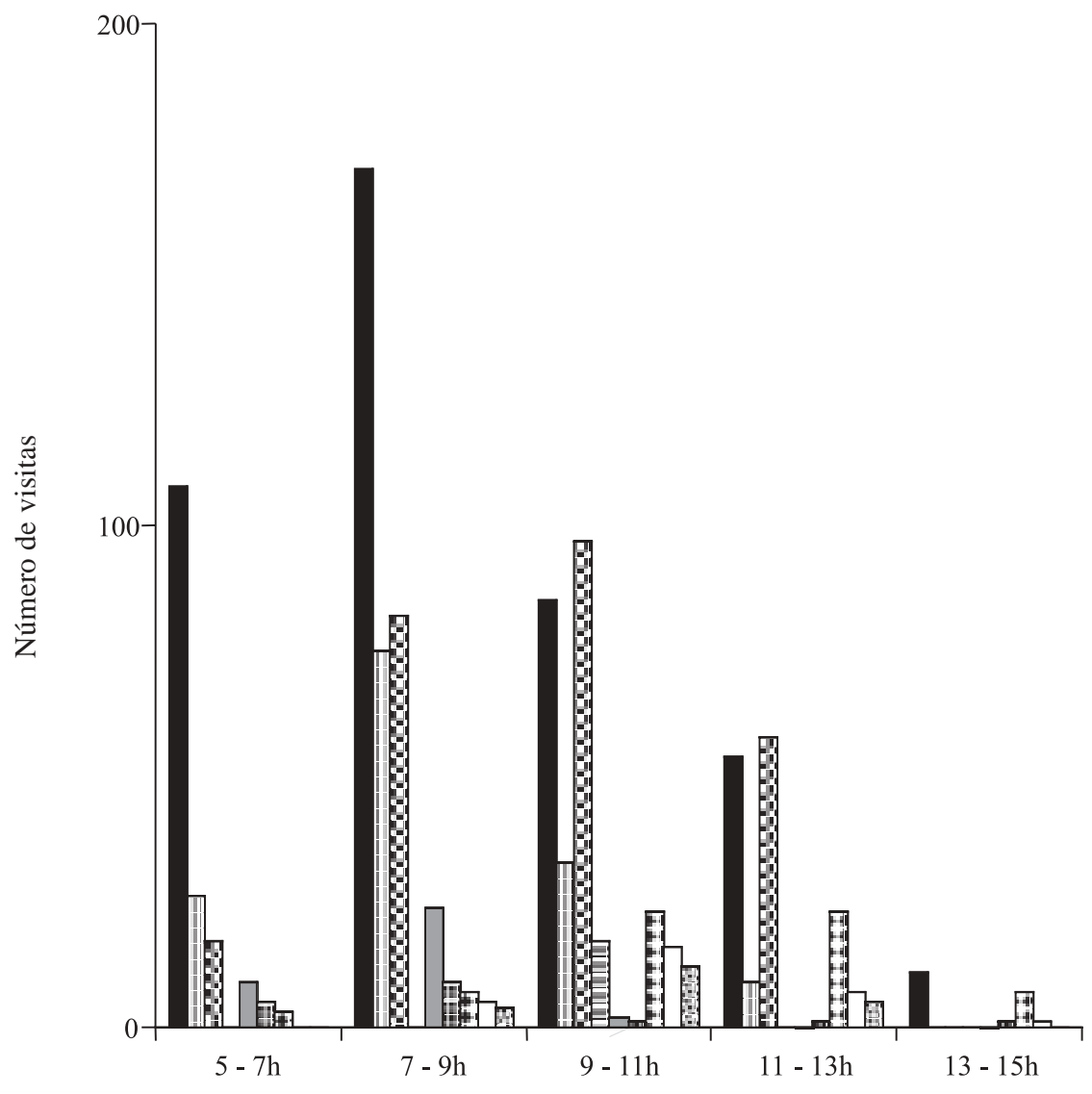

Horas

Figura 4. Média de freqüência dos visitantes florais de Solanum stramonifolium em um dia de visita ( $\mathrm{n}=8$ dias). - Melipona scutellaris; $\square$ Eufriesea surinamensis; Xylocopa suspecta; $\boxminus$ Bombus brevivilus; $\square$ Euglossa sp.; 田 Paratetrapedia sp., $Z$ Halictidae; $\square$ Eulaema cingulata, , Eulaema nigrita. 

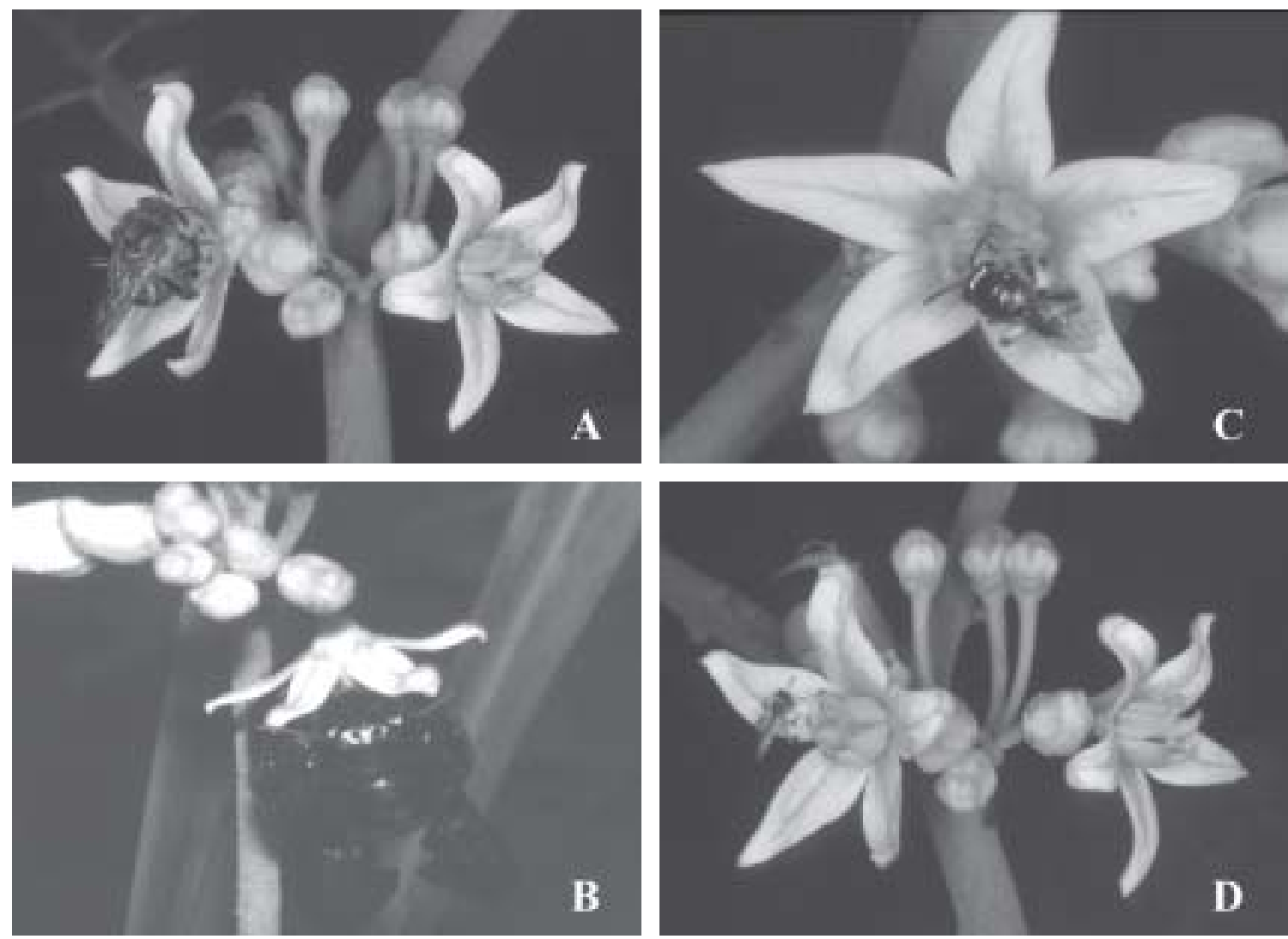

Figura 5. Visitantes florais de Solanum stramonifolium. A) Melipona scutellaris; B) Xylocopa suspecta; C) Pseudoaugochloropsis sp., vibrando apenas uma antera; D) Tetragonisca angustula, um dos pilhadores de S. stramonifolium.

antera e curvam seu tórax sobre os poros (Fig. 5C). A vibração é feita antera por antera, fazendo com que a duração de suas visitas em cada flor seja bem maior quando comparado com as abelhas maiores. Os representantes da família Halictidae são polinizadores mais eficientes nas flores pequenas, nas quais conseguem vibrar todo o cone de anteras e tocar o estigma das flores hermafroditas. Nas flores maiores, contudo, o contato com o estigma é rápido, ocorrendo apenas durante o deslocamento pelas anteras. Mesmo sendo uma abelha vibradora, Paratetrapedia sp. geralmente não poliniza as flores de S. stramonifolium devido ao seu pequeno tamanho. A remoção de pólen do tórax para as corbículas se dá na própria flor; para isto, a abelha segura-se com as pernas anteriores na antera e se limpa com as medianas e posteriores.

Logo após a ação das abelhas vibradoras, o pólen residual da corola e do ápice das anteras era coletado pelas pequenas abelhas pilhadoras Plebeia sp. e Tetragonisca angustula (Fig. 5D). A coleta de pólen por Trigona sp. era feita através de cortes efetuados nas anteras com as mandíbulas.

Como descrito por Coleman \& Coleman (1982), a diminuição do tamanho da abelha também reduz a sua eficiência como polinizador de espécies de Solanum, pois torna-se mais raro o seu contato com o estigma, o que faz com que esta espécie se enquadre no grupo de flores 
polinizadas por abelhas grandes $(\geq 12 \mathrm{~mm}$ compr.), proposto por Frankie et al. (1983).

Apesar de Augochloropsis sp. e Pseudoaugochloropsis sp. apresentarem o mesmo comprimento de Melipona scutellaris, elas não são consideradas polinizadores efetivos pelo fato de não conseguirem vibrar todo o cone de anteras ao mesmo tempo, sendo, portanto, polinizadores ocasionais que contactam o estigma rapidamente durante seu deslocamento pelas anteras.

Apesar da deiscência poricida, de certo modo, restringir a variedade dos visitantes florais a apenas um grupo reduzido de abelhas, que conseguem vibrar seus músculos indiretos de vôo, esse sistema reduz a perda de pólen, visto que há um direcionamento do pólen expelido, através da vibração, para partes bem definidas do corpo do polinizador (Buchmann 1983). Porém, nas primeiras visitas, é visível a perda de quantidade considerável de grãos ao ser expelida a nuvem de pólen das anteras. A deiscência poricida das anteras faz com que Solanum stramonifolium selecione uma guilda de polinizadores hábeis na coleta do recurso; e a abundância de flores e a grande quantidade de pólen promovem a constância das abelhas, garantindo assim a sua reprodução.

\section{Agradecimentos}

À Sílvia Regina A. Santos, pelo auxílio no trabalho de campo; aos Drs. Clemens Schlindwein e Márcio Oliveira, pela identificação das abelhas; à Direção do Parque Estadual de Dois Irmãos, ela permissão para realização do trabalho na área do Açude do Prata; ao CNPq, pelo auxílio financeiro.

\section{Referências bibliográficas}

Avanzi, M. R. \& Campos, M. J. O. 1997. Estrutura de guildas de polinização de Solanum aculeatissimum Jacq. e S. variable Mart. (Solanaceae). Revista Brasileira de Biologia 57: 247-256.
Bernhardt, P. 1996. Anther adaptation in animal pollination. Pp. 192-220. In: W. G. D' Arcy \& R. C. Keathing (Eds.). The anther. Cambridge University Press.

Bowers, K. A. W. 1975. The pollination ecology of Solanum rostratum (Solanaceae). American Journal of Botany 62: 633-638.

Buchmann, S. L.; Jones, C. E. \& Colin, L. J. 1977. Vibratile pollination of Solanum douglasii and Solanum xantii (Solanaceae) in Southern California. The Wasman Journal Biology 35: 125.

Buchmann, S. L. 1983. Buzz pollination in Angiosperms. Pp. 73-113. In: C.E. Jones \& R.J. Little (Eds.). Handbook of experimental pollination biology. Van Nostrand \& Reinhold, New York.

Coleman, J. R. \& Coleman, M. A. 1982. Reproductive biology of andromonoecius Solanum (Solanum palinacanthum Dunal). Biotropica 14: 69-75.

Dafni, A. 1992. Pollination ecology. Oxford University Press, Oxford.

D’Arcy, W. G. 1973. Flora of Panama. Fam. Solanaceae. Annals of the Missouri Botanical Garden 60: 573-780.

Dukas, R. \& Dafni, A. 1990. Buzz-pollination in three nectariferous Boraginaceae and possible evolution of buzz-pollinated flowers. Plant Systematics and Evolution. 169: 65-68.

Endress, P. K. 1994. Diversity and evolutionary biology of tropical flowers. Cambridge University Press, Cambridge.

Faegri, K. \& van der Pijl, L. 1979. The principles of pollination ecology. $3^{\text {a }}$ ed. Pergamon Press, London.

Frankie, G. W.; Haber, W. A.; Opler, P. A. \& Bawa, K. S. 1983. Characteristics and organization of large bee pollination systems in the Costa Rican dry forest. Pp.411-447. In: C. E. Jones, \& R. J. Little, (Eds.). Handbook of experimental pollination biology. Van Nostrand \& Reinhold, New York.

Gentry, A. H. 1974. Flowering phenology and diversity in tropical Bignoniaceae. Biotropica 6: 64-68.

Harris, J. A. \& Kucks, O. M. 1902. Observations of the pollination ecology of Solanum rostratum Dunal and Cassia chamaecrista L. University of Kansas Science Bulletin 1: 15-43.

Kearns, C. A. \& Inouye, D.W. 1993. Techniques for pollination biologists. Colorado University Press, Colorado. 
Linsley, E. G. 1962. The colletid Ptiloglossa arizonensis Timberlake, a matinal pollinator of Solanum. Pan-Pacific Entomology 38: 75-82.

Linsley, E. G. \& Cazier, M. A. 1963. Further observations on bees which take pollen from plants of the genus Solanum. Pan-Pacific Entomology 39: 1-18.

Lopes, A. V. \& Machado, I. C. S. 1986. Biologia floral de Swartzia pickelii Killip ex Ducke (Leguminosae-Papilionoideae) e sua polinização por Eulaema spp. (Apidae-Euglossini). Revista Brasileira de Botânica 1: 17-24.

Machado, I. C.; Lopes, A. V. \& Pôrto, K. C. (Orgs.) 1998. Reserva Ecológica de Dois Irmãos: Estudos em um remanescente de Mata Atlântica em área urbana. SECTMA (Secretaria da Ciência, Tecnologia e Meio Ambiente). Editora Universitária -UFPE, Recife.

Michener, C. D. 1962. An interesting method of polen collecting by bees from flowers with tubular anthers. Revista de Biologia Tropical 10: 167-175.

Moura, R. A.; Wada, C. S.; Purchio, A. \& Almeida, T.V. 1987. Técnicas de laboratório. Atheneu, Rio de Janeiro.

Newstrom, L.E.; Frankie, G.W. \& Baker, H.G. 1994. A new classification for plant phenology based on flowering patterns in lowland tropical rain forest trees at La Selva, Costa Rica. Biotropica 26: 141-159.
Oliveira-Filho, A.T. \& Oliveira, L.C.A. 1988. Biologia floral de uma população de Solanum lycocarpum St. Hil. (Solanaceae) em Lavras, MG. Revista Brasileira de Botânica 11: 23-32.

Roubik, D.W. 1992. Ecology and natural history of tropical bees. Cambridge Tropical Biology series. Cambridge University Press, USA.

Schilling Jr., E. E. \& Heiser Jr., C. B. 1979. Crossing relationships among diploid species of the Solanum nigrum complex in North America. American Journal of Botany 66: 709-716.

Storti, E.F. 1988. Biologia floral de Solanum sessiliflorum Dun. var. sessiliflorum, na região de Manaus, AM. Acta Amazonica 18: 55-65.

Symon, D.E. 1979. Sex forms in Solanum (Solanaceae) and the role of pollen collecting insects. Pp.385398. In: J. G. Hawkes; R. N. Lester, \& A. D. Skelding, (Eds.). The biology and taxonomy of the Solanaceae. Academic Press, London.

Vogel, S. 1978. Evolutionary shifts from reward to deception in pollen flowers. Pp. 89-96. In: A. J. Richards (Ed.) The pollination of the flowers by insects. Academic Press, London.

Vogel, S. 1990. The role of scent glands in pollination. Smithsonian Institution Libraries and The National Science Foundation, Washington, D. C.

Wille, A. 1963. Behavioural adaptations of bees for pollen collecting from Cassia flowers. Revista de Biologia Tropical 11: 205-210. 